\title{
Polar cap index comparisons with AMIE cross polar cap potential, electric field, and polar cap area
}

\author{
A. J. Ridley ${ }^{1}$ and E. A. Kihn ${ }^{2}$ \\ Received 19 November 2003; revised 2 February 2004; accepted 4 March 2004; published 2 April 2004.
}

[1] The polar cap index (PCI) has been shown to be quite useful as an index of the electrodynamic processes within the high-latitude ionosphere. Here we use between 150 and 180 magnetometers to model the high-latitude electrodynamics with the assimilative mapping of ionospheric electrodynamics (AMIE) technique for all of 1997-2001. We compare AMIE calculations of the cross polar cap potential (CPCP), polar cap electric field, and polar cap area to the Northern PCI and the existing PCIbased relationships for these quantities. We find that the existing PCI estimates underestimate all of the AMIE derived quantities. The PCI is best correlated with the CPCP, while it correlates least with the polar cap electric field. In addition, there is a seasonal effect in the relationship between all AMIE quantities and the PCI. It is most pronounced in the electric field. The polar cap area is shown to have a linear fit in the summer and an asymptotic fit in the winter. These differences indicate that there may be a conductance difference in the techniques. We derive new relationships between the Northern PCI and the AMIE derived polar cap area, CPCP, and polar cap electric field which include the seasonal dependence. INDEX TERMS: 2407 Ionosphere: Auroral ionosphere (2704); 2411 Ionosphere: Electric fields (2712); 2447 Ionosphere: Modeling and forecasting; 2475 Ionosphere: Polar cap ionosphere. Citation: Ridley, A. J., and E. A. Kihn (2004), Polar cap index comparisons with AMIE cross polar cap potential, electric field, and polar cap area, Geophys. Res. Lett., 31, L07801, doi:10.1029/2003GL019113.

\section{Introduction}

[2] There are a number of global indices that are used to indicate the state of the magnetosphere at any given time. For example, the auroral electrojet index is meant to describe the maximum amount of auroral activity occurring (or maximum westward current flowing) within a certain magnetic latitude band. The $D_{s t}$ index indicates the strength of the magnetospheric ring current (plus some addition of magnetopause and cross-tail currents). The polar cap index (PCI) indicates the amount of energy which the solar wind and interplanetary magnetic field (IMF) are transferring to the magnetosphere through direct driving (i.e., merging) [Troshichev et al., 1988, 1996b]. The polar cap index is derived from a single station per hemisphere. There are therefore 2 independent polar cap indices - one derived from

\footnotetext{
${ }^{1}$ Center for Space Environment Research, University of Michigan, Ann Arbor, Michigan, USA.

${ }^{2}$ National Geophysical Data Center, Boulder, Colorado, USA.
}

the Thule magnetometer (Northern) and one from the Vostok magnetometer (Southern). The PCI is continuous from the present day back to 1975 . Recently, it has been shown that the Northern and Southern polar cap indices can differ systematically [Lukianova et al., 2002]. All calculations within this study are conducted using the Northern PCI computed by the Danish Meteorological Institute (DMI).

[3] The assimilative mapping of ionospheric electrodynamics (AMIE) technique [Richmond and Kamide, 1988] has been used in a number of studies to relate the highlatitude ionospheric electrodynamics to the IMF. AMIE is a difficult technique to use because of the large amount of data needed to create a coherent and accurate solution. In addition, the data is distributed among many providers and the quality is quite variable. Therefore, creating proxies of the electrodynamics parameters that can be computed from AMIE can be quite useful. For example, Chun et al. [1999] calculated a relationship between the PCI and AMIE derived hemispheric integrated Joule heating.

[4] Troshichev et al. [1996a] relates the PCI to CPCP and the radius of the polar cap in the morning and evening sectors. These relationships were determined from 6 months (January-June 1990) of Akebono satellite data, composed of 325 orbits. Troshichev et al. [2000] relate the PCI to the "near-pole" electric field. This was done using 450 polar passes of DMSP during days that included low and high activity. They related the PCI to the electric field (in $\mathrm{mV} / \mathrm{m}$ ) near the magnetic pole, and determined the following relationship:

$$
E=-0.29 P C I^{2}+7.65 P C I+15.3
$$

for the Northern PCI. This is equation (2) from Troshichev et al. [2000] multiplied by 1.7, as specified in the paper, assuming an ionospheric altitude of $110 \mathrm{~km}$, as AMIE does.

\section{Technique}

[5] The AMIE technique was run for every minute of 1997-2001 for both Northern and Southern hemispheres using between 150-180 magnetometers spaced nonuniformly across the Earth. For this study, we concentrate on the Northern hemisphere, which included the vast majority of the magnetometers. 2.6 million calculations of the high-latitude electrodynamics, including the potential, electric field, conductances, auroral precipitation, and horizontal and field-aligned currents were done. From this database, the CPCP, maximum polar cap electric field magnitude (i.e., largest electric field poleward of $80^{\circ}$ ), and the polar cap area were extracted. The polar cap area was calculated from the auroral precipitation results 
in a similar manner as done in Baker et al. [2000]: the location of the maximum total electron energy flux was determined at each MLT (i.e., 24 wedges); then move towards the pole until the precipitation flux has fallen below $25 \%$ of the maximum value; then calculate the area poleward of this location. The $25 \%$ cut-off was found by Baker et al. [2000] to be very effective in finding the open/closed field-line boundary using total electron precipitation energy flux derived from POLAR images of the aurora. Kihn and Ridley (E. Kihn and A. Ridley, A statistical analysis of the AMIE auroral specification, submitted to Journal of Geophysical Research, 2004) have shown that, statistically, AMIE puts the location of the peak of the auroral oval within 1 grid cell (i.e., $2^{\circ}$ ) of satellite observations of the peak. This implies that AMIE can reproduce the location of the oval relatively well.

[6] AMIE includes both solar and auroral driven conductance background models. The solar conductance is specified by a solar zenith angle and was developed using data from Chatanika radar. For different solar zenith angles $(\zeta)$, the conductance is:

$$
\begin{gathered}
\Sigma_{H}=1.8 \cos (\zeta) F_{10.7}^{1 / 2} \quad \zeta<65^{\circ} \\
\Sigma_{H}=\Sigma_{H}\left(65^{\circ}\right)-0.27\left(\zeta-65^{\circ}\right) \quad \zeta>65^{\circ} \\
\Sigma_{P}=0.5 \cos \left(\zeta^{2 / 3}\right) F_{10.7}^{2 / 3} \quad \zeta<65^{\circ} \\
\Sigma_{P}=\Sigma_{P}\left(65^{\circ}\right)-0.24\left(\zeta-65^{\circ}\right) \quad \zeta>65^{\circ} \\
\Sigma_{P}=\Sigma_{P}\left(100^{\circ}\right)-0.13\left(\zeta-100^{\circ}\right) \quad \zeta>100^{\circ}
\end{gathered}
$$

where $\Sigma_{H}$ and $\Sigma_{P}$ are the Hall and Pedersen conductance, respectively, and $F_{10.7}$ is the solar radio flux. $\Sigma_{H}\left(\Sigma_{P}\right)$ is limited to a minimum value of 0.8 (0.4) mho. The conductance is then modified according to the local magnetic field, as described by Rasmussen et al. [1988] and Figure 9 in Kamide and Matsushita [1979].

[7] AMIE modifies the conductances using the formulation given by Ahn et al. [1998], which relates the groundbased magnetic perturbations to the conductance and electron precipitation. At each magnetometer location, the magnetic perturbation is converted to a Hall and Pedersen conductance. The solar conductance is subtracted from this (if the station is on the day-side). The Robinson et al. [1987] formulation is then used to convert the conductances (at each station) to total and average electron precipitation. These individual data sources are then used to derive a particle precipitation pattern (used to calculate the polar cap area). The Robinson et al. [1987] formulation is used once again to derive auroral driven Hall and Pedersen conductances. These are added to the solar conductances to derive total conductances. The Fuller-Rowell and Evans [1987] (particles) and Weimer [1996] (potentials) models were used as background patterns for all runs.

[8] The cross polar cap potential and electric field were calculated from the PCI on a 15 minute time-scale, using equations (3) and (4). Troshichev et al. [1996a] provided the dawn and dusk radius of the polar cap. A more physically meaningful quantity is the area of the polar cap, since it indicates the total amount of magnetic flux (and therefore
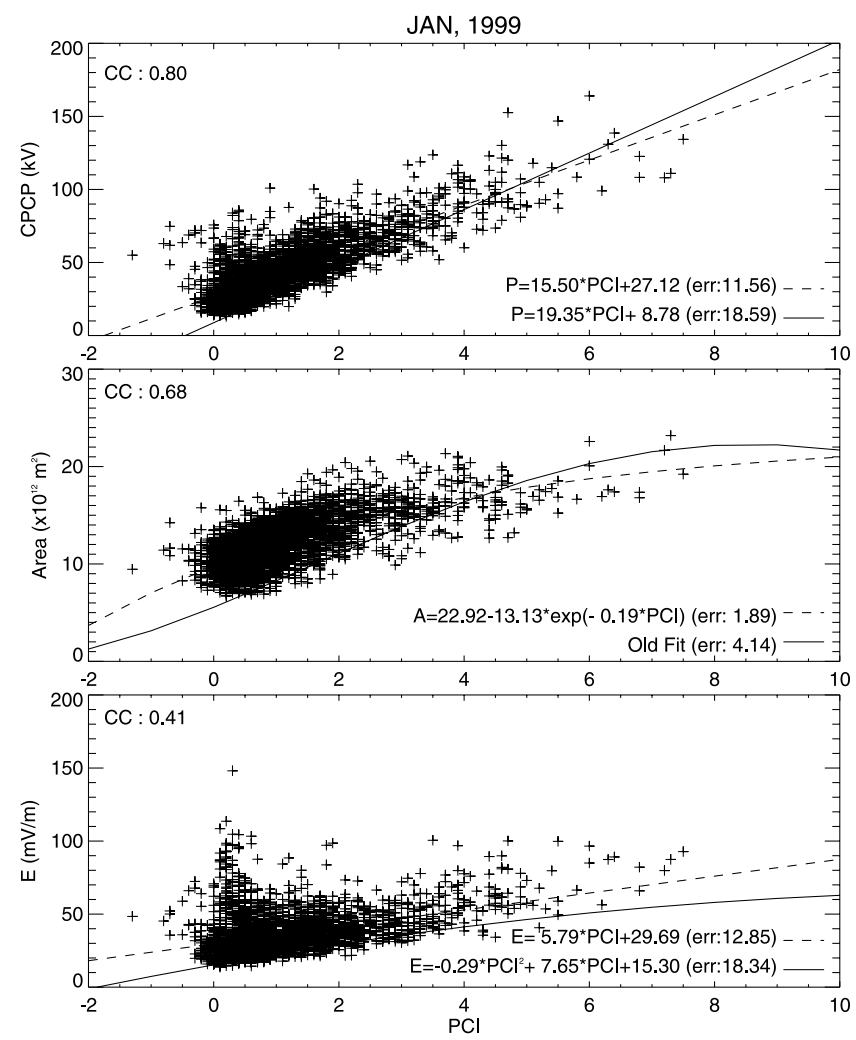

Figure 1. The PCI versus the AMIE derived CPCP, $A_{p c}$, and $E_{p c}$ (from top to bottom) for Jan., 1999. The solid lines are the relationships in Troshichev et al. [1996a, 2000], while the dashed lines are fits which are described here. The correlation coefficient (CC) reported is between the AMIE derived quantities and the PCI. The errors (err) are the rootmean squared differences between the predicted PCI-based quantities and the AMIE derived quantities.

energy) in the lobes. We can therefore calculate the area from the dawn and dusk radii:

$$
A_{P C}=\pi\left[\frac{1}{4} R_{a m}^{2}+\frac{1}{4} R_{p m}^{2}+\frac{1}{2}\left(\frac{R_{a m}+R_{p m}}{2}\right)^{2}\right]
$$

where $A_{P C}$ is the area of the polar cap, and $R_{a m}$ and $R_{p m}$ are radius of the polar cap in the dawn and dusk sectors (as defined by Troshichev et al. [1996a]), but converted to meters. The above formula assumes 4 wedges with the following radii: (1) $R_{a m}$, (2) $R_{p m}$, and (3) two with $\left(R_{a m}+\right.$ $\left.R_{p m}\right) / 2$. The AMIE CPCP, area, and electric field were averaged over 15 minutes to match the time of the PCI computed values.

\section{Results}

[9] Figure 1 shows comparisons between the PCI and AMIE computed potential, polar cap area, and electric field for January, 1999. The solid lines on each plot indicate the relationships in Troshichev et al. [1996a, 2000]. These lines follow the general trends observed in the AMIE results, but there are some systematic differences. For example, the relationship under-predicts the 


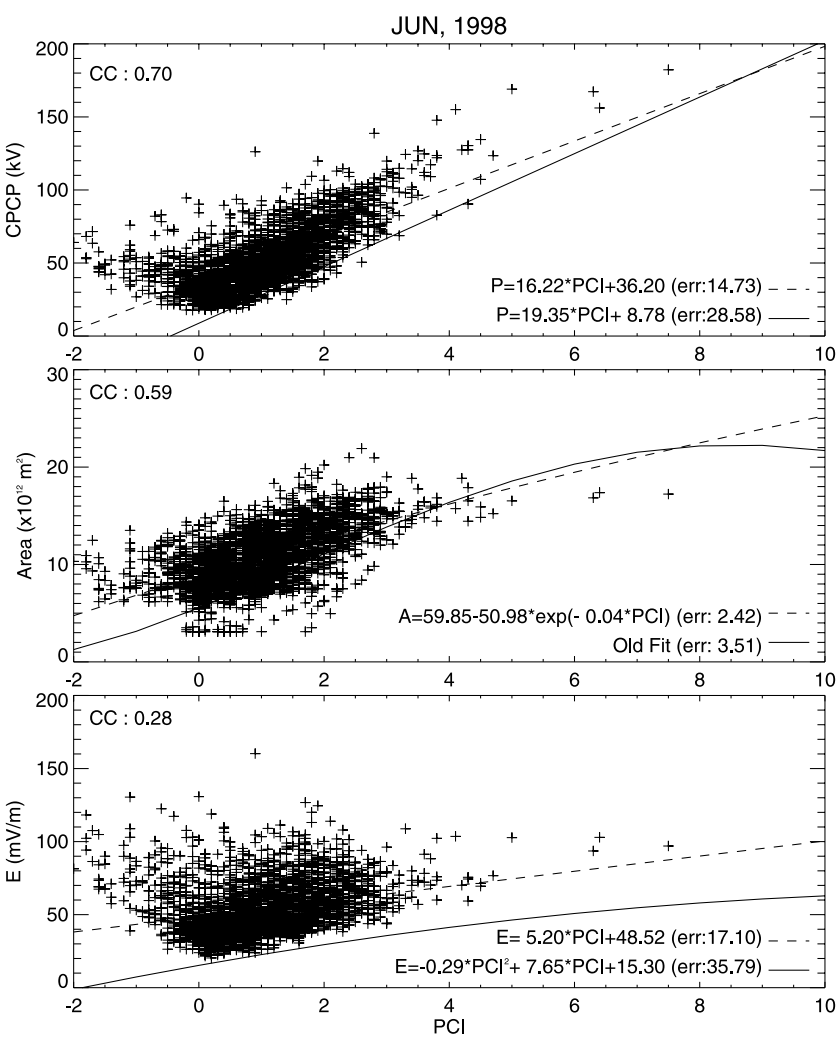

Figure 2. June 1998 in the same format as Figure 1.

polar cap electric fields for almost all PCI values. The formulation also under-predicts both the $\mathrm{CPCP}$ and polar cap area at low values of PCI, while doing quite well at high values of PCI. Another issue is that the Troshichev et al. [2000] formulation of the CPCP becomes negative at around PCI $=-0.5$, which is unphysical. Finally, the PCI predicted polar cap area starts to decrease at around PCI $=9$, which is not typically observed.

[10] For these reasons, new fits were calculated for the three quantities. The dashed line shows the new fits. For the $\mathrm{CPCP}$ and the polar cap electric field, linear relationships were determined, while an exponential fit to the polar cap area worked best. The following relationships were determined for January 1999:

$$
\begin{gathered}
\Phi=27.12+15.5 P C I \\
A_{P C}=22.93-13.13 e^{-0.19 P C I} \\
E_{P C}=29.69+5.79 P C I .
\end{gathered}
$$

[11] Figure 2 shows the relationship between the PCI index and the AMIE derived quantities for June, 1998. There are some differences between this and the January 1999 time period. For example, the PCI index is much more confined below 4, while the AMIE electric field and CPCP contain a similar range of values as the January 1999 period. Because of this, the cross correlation coefficients are all reduced. The fits for June, 1998 are:

$$
\begin{gathered}
\Phi=38.20+16.22 P C I \\
A_{P C}=59.85-50.98 e^{-0.04 P C I} \\
E_{P C}=48.52+5.20 P C I .
\end{gathered}
$$

One of the largest differences is in the exponential fit to the area. The asymptotic limit of the area is almost $60 \times$ $10^{12} \mathrm{~m}$, which is over a factor of 2 larger than the asymptotic limit for January 1999. Complementary with that is the e-folding factor, which is 5 times smaller than the January 1999 factor. These two combined imply that the area during June 1998 is approximately linearly related to the PCI, while the area during January 1999 is exponentially related to the PCI. During June 1998, both the electric field and potential have larger y-intercepts (but smaller slopes) than during January 1999, implying that for lower polar cap indices, the electric field and potential will be larger in June than in January; and for large PCI, the values will be similar or smaller in June than in January.

[12] Because there is such a large difference between a summer and a winter period, we examine the correlation and fit coefficients as a function of the month of the year. Figure 3a shows the trend in the relationship between the AMIE derived CPCP and the PCI. In the top plot, there is a trend for the correlation coefficient to peak in the winter months and reach minimal values in the summer months. When a sin function is fit through these values, the peak to $\mathrm{a}$

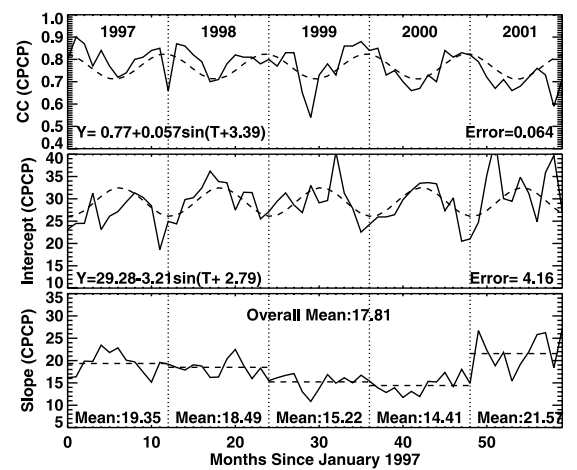

b

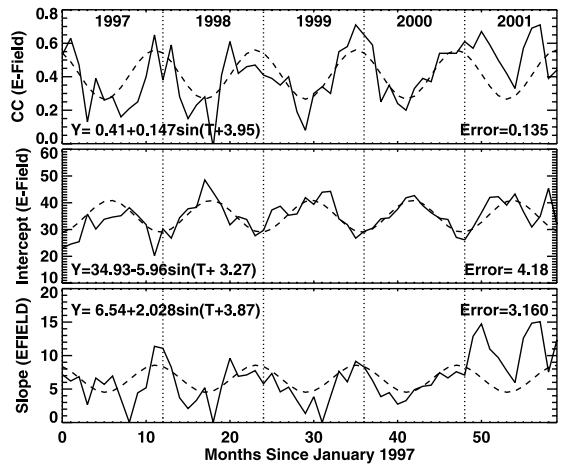

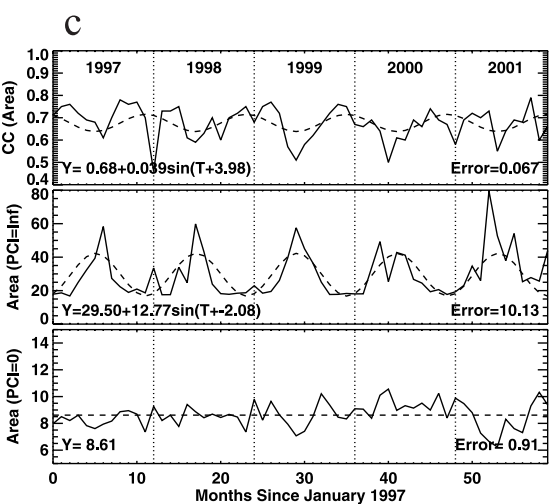

Figure 3. (a) The monthly AMIE CPCP vs. PCI CC, fitted intercepts and slopes from 1997 through 2001 (from top to bottom). Fits through these are shown as dashed lines. (b) The monthly AMIE $E_{p c}$ vs. PCI CC, fitted intercepts and slopes (from top to bottom). (c) The top plot is the monthly AMIE $A_{p c}$ vs. PCI CC, while the middle and bottom plots show the asymptotic limit of the area (i.e., $\mathrm{PCI}=\infty$ ) and the baseline area (i.e., $\mathrm{PCI}=0$ ). 
peak differences is $14 \%$ of the mean value, which is relatively small. A similar trend is observed in the intercept between the PCI and the CPCP, with a minimum during the winter months and a maximum during the summer. The peak to peak deviation is about $23 \%$. The slope shows some indication of a seasonal variation, but the year to year variation in the mean is more significant. One may conclude that the CPCP may be related to the AMIE derived CPCP with a seasonally dependent $y$-intercept:

$$
\Phi=29.28-3.31 \sin (T+1.49)+17.81 P C I,
$$

where $T$ is the month of the year normalized to $2 \pi$ (i.e., Jan. $=0$, July $=6 * 2 * \pi / 12=\pi$, Dec. $=11 * 2 * \pi / 12$ ). This formula can be used in general for a relationship between the Northern PCI and the ionospheric CPCP.

[13] Figure 3b shows the fit and correlation coefficients for each month from 1997 through 2001 for the AMIE derived polar cap electric field. Each of these show a seasonal variation, such that the electric field can be related to the PCI through the relationship:

$E_{P C}=34.87-5.67 \sin (T+3.21)$

$$
+[6.58+2.04 \sin (T+2.03)] P C I \text {. }
$$

The peak to peak variation in the slope is $62 \%$, which is caused by the lack of correlation between the PCI and the electric field during the summer months. The y-intercept varies by $32.5 \%$ during the year, which is to compensate for the lack of slope in the summer months.

[14] Figure 3c shows the monthly relationships for the area with the PCI. Since the fits are with an exponential, the second and third plots are different than Figures $3 \mathrm{a}$ and $3 \mathrm{~b}$. These two plots show the asymptotic limit of the area (i.e., PCI $=\infty$, or the first numbers in equations (9) and (13)) and the baseline area (i.e., PCI $=0$, or the first + second number in equations (9) and (13). There are few variations in the baseline area with no seasonal dependence being observed. The asymptotic value, on the other hand, varies significantly (as does the exponent, which is not shown). The strong variation in the exponent and asymptotic value indicates that the fit is switching between a linear and non-linear relationship for summer and winter, respectively. This is seen when the plots in Figure 1 are compared - the fit in January is an exponential, while the fit in June is linear over the entire range of PCI values. The relationship between the polar cap area and the PCI can be described as:

$$
A_{P C}=29.72-21.11 e^{\beta}+12.73 \sin (T-1.08)\left(1-e^{\beta}\right)
$$

where $\beta=(-0.24+0.16 \sin (T-1.08)) P C I$.

\section{Summary and Conclusions}

[15] We have run the AMIE technique for all of 1997 through 2001 using 150-180 ground-based magnetometers on a 1 minute cadence. The results of the cross polar cap potential, polar cap electric field, and polar cap area were determined. This study presents a comparison between the AMIE results and the polar cap index. We find that the current formulation which relates the CPCP, E-field, and area to the PCI to be lacking in a number of regards, but adequate in some circumstances. Because of the limitations, we rederive the relationships.

[16] When the newly derived relationships are examined on a month by month basis, a clear seasonal dependence is observed. This dependence is quite strong in the relationship between the polar cap electric field and the PCI. It exists in the relationship between the CPCP, but is much weaker. In the relationship between the PCI and the polar cap area, the seasonal dependence is on whether the relationship is linear or exponential, while the base area (i.e., when $\mathrm{PCI}=0$ ) shows no sign of a seasonal dependence.

[17] Both the AMIE and the PCI take the season into account, but the seasonal dependence between the two shows that they are not consistent with each other. As described above, AMIE takes the solar driven conductance into account. Different solar driven conductance models could be used in AMIE to determine the effects.

[18] The study by Lukianova et al. [2002] shows that there is a seasonal dependence between the Northern (provided by DMI) and Southern PCI when the PCI is large. They also show that the Northern PCI is typically weaker than the Southern PCI, which matches our assessment that the PCI formulations given by Troshichev et al. [1996a, 2000] predict values that are too low.

[19] It would be of further use to correlate the PCI and AMIE derived values for many years with a conductance independent source, such as the SuperDARN or incoherent scatter radar networks, or DMSP satellites. Any seasonal variation observed between AMIE or PCI and a conductance independent source could be corrected by improving the solar conductance model within AMIE or the method for computing PCI.

[20] Acknowledgments. Research at the University of Michigan was supported by NSF grant ATM-0077555. The following data was used in AMIE: CANOPUS (Canadian Space Agency), IMAGE (Finnish Meteorological Institute), Measure (University of California Los Angles), Greenland coastal chains (Danish Meteorological Institute), MAGIC (University of Michigan), MACCS (Augsburg and Boston University), 210 magnetic meridian (Kyushu University and Nagoya University), and Intermagnet.

\section{References}

Ahn, B.-H., A. Richmond, Y. Kamide, H. Kroehl, B. Emery, O. de la Beaujardiére, and S.-I. Akasofu (1998), An ionospheric conductance model based on ground magnetic disturbance data, J. Geophys. Res., $103,14,769$.

Baker, J., C. Clauer, A. Ridley, and V. Papitashvili (2000), The nightside poleward boundary of the auroral oval as seen by the DMSP and the Ultraviolet Imager, J. Geophys. Res., 105, 21,267.

Chun, F. K., D. J. Knipp, M. G. McHarg, G. Lu, B. A. Emery, S. Vennerstrom, and O. A. Troshichev (1999), Polar cap index as a proxy for hemispheric joule heating, Geophys. Res. Lett., 26, 1101.

Fuller-Rowell, T., and D. Evans (1987), Height-integrated Pedersen and Hall conductivity patterns inferred from TIROS-NOAA satellite data, J. Geophys. Res., 92, 7606.

Kamide, Y., and S. Matsushita (1979), Simulation studies of ionospheric electric fields and currents in relation to field-aligned currents: 1. Quiet periods, J. Geophys. Res., 84, 4083.

Lukianova, R., O. Troshichev, and G. Lu (2002), The polar cap magnetic activity indices in the southern (PCS) and northern $(\mathrm{PCN})$ polar caps: Consistency and discrepancy, Geophys. Res. Lett., 29(18), 1879, doi:10.1029/2002GL015179.

Rasmussen, C., R. Schunk, and V. Wickwar (1988), A photochemical equilibrium model for ionospheric conductivity, J. Geophys. Res., 93, 9831.

Richmond, A., and Y. Kamide (1988), Mapping electrodynamic features of the high-latitude ionosphere from localized observations: Technique, J. Geophys. Res., 93, 5741. 
Robinson, R., R. Vondrak, K. Miller, T. Dabbs, and D. Hardy (1987), On calculating ionospheric conductances from the flux and energy of precipitating electrons, J. Geophys. Res., 92, 2565.

Troshichev, O., V. Andezen, S. Vennerstrom, and E. Friis-Cristensen (1988), Magnetic activity in the polar cap: A new index, Planet. Space Sci., 36, 1095.

Troshichev, O., H. Hayakawa, A. Matsuoka, T. Mukai, and K. Tsuruda (1996a), Cross polar cap diameter and voltage as a function of $P C$ index and interplanetary quantities, J. Geophys. Res., 101, 13,429.

Troshichev, O., E. Shishkina, C.-I. Meng, and P. Newell (1996b), Identification of the poleward boundary of the auroral oval using characteristics of ion precipitation, J. Geophys. Res., 101, 5035.
Troshichev, O. A., R. Y. Lukianova, V. O. Papitashvili, F. J. Rich, and O. Rasumussen (2000), Polar cap index (PC) as a proxy for ionospheric electric field in the near-pole region, Geophys. Res. Lett., 27, 3809.

Weimer, D. (1996), A flexible, IMF dependent model of high-latitude electric potential having "space weather" applications, Geophys. Res. Lett., $23,2549$.

E. A. Kihn, National Geophysical Data Center, Boulder, CO, USA.

A. J. Ridley, Center for Space Environment Research, University of Michigan, Ann Arbor, MI 48109-2143, USA. (ridley@umich.edu) 